\title{
Research on the Analysis of Muscle Fatigue Based on the Algorithm of Wavelet Packet Entropy in sEMG
}

\author{
Bowen Zhang ${ }^{\mathrm{a}}$, Bingdie Huang ${ }^{\mathrm{a}}$, Qun Wu ${ }^{\mathrm{b}, 1}$, Guowei $\mathrm{Lu}^{\mathrm{c}}$, Yao $\mathrm{Wu}^{\mathrm{a}}$ \\ ${ }^{a}$ Wenzhou Business College, Wenzhou, 325000, China \\ ${ }^{b}$ Zhejiang Sci-Tech University, Hangzhou, 310018, China \\ ${ }^{c}$ Zhejiang College of security technology, Wenzhou, 325000, China
}

\begin{abstract}
Surface Electromyography (sEMG) has been widely applied in different fields, such as human-computer interaction and medical rehabilitation. This paper deeply studies the theoretical research of muscle fatigue analysis and scientific experiments of sEMG, especially related to muscle fatigue. It provides a theoretical basis for not only building an assessment method of muscle fatigue but also testing the relationship between the value of wavelet packet entropy and the complexity of signal frequency. Additionally, the field value of muscle fatigue has been uncovered. In this research, the experiment tests the energy variation of wavelet packet entropy when testing the muscle contraction by the sEMG signals of brachioradialis. It means that the experiment decomposes frequency band, and then $\mathrm{SEMG}$ is analyzed by the entropy and distribution of energy. The result of experiment indicated that the index of wavelet packet entropy has an efficient and quick performance on analyzing complexity of signal system. In the experiment of muscle fatigue, wavelet packet entropy can present high accuracy, instant reaction, stronger consistency and reliability, which is significant for the achievement of the real-time monitoring and clinical research of bioelectrical signals.
\end{abstract}

Keywords. muscle fatigue, wavelet packet entropy, recognition of muscle pattern, medical rehabilitation

\section{Introduction}

Surface Electromyography (sEMG) is a bioelectric signal generated by muscle contraction which not only reveals the physical and status of neuromuscular system but also benefits for invasive signal collection. [1] [2]. It is the widest application of study in assessing muscle fatigue and can be used for other fields, such as sports, rehabilitation, and human-computer interaction. [3] However, due to the huge individual difference and strong subjectivity of muscle fatigue, there is no common standard for the assessment of muscle fatigue. [4] Therefore, assessing muscle fatigue has great significance in terms of further treatment and later treatment effects [5].

With the deeper study of muscle fatigue, experts have found plenty of sEMG characteristic parameters that could be used for assessment of muscle fatigue, such as

${ }^{1}$ Corresponding Author: Qun Wu, Postal address: Zhejiang Sci-Tech University, Hangzhou, China,310018; Email: wuq@zstu.edu.cn. 
rating of perceived exertion (RPE) near-infrared spectroscopy (NIRS), mechanomyogramphy(MMG), electromyography (EMG) and so on. [6] 1-Mullaetal.(2011) revealed the classification of fatigue level by pseudo wavelet. His experiment proved that using pseudo wavelet had better effects on estimating the level of muscle fatigue than traditional wavelet; Rogers and Macisaac (2013) compared five methods of estimating muscle fatigue, including PCA (Principal Component Analysis), GMI (Generalized Mapping Index), NSM (Normalized Spectral Moments), WI(Wavelet based Indices), HE(fractal -based Hurst Exponent) and concluded PCA surpass than other four methods. [7] [8] To find the method of improving the accuracy of predicting muscle fatigue, several experts applied related complex math models in the study. Mobasseret (2007) concluded FOS has better performance on the prediction accuracy and training efficiency; Hashemietal et al. [9] (2010) illustrated that PCI was more accurate than FOS in terms of estimation of the muscle strength of the upper arm muscles in dynamic elbow joint exercises; Stauden-mannetal et al. used PCA to estimate the level of muscle activation for the prediction of muscle power. [10] However, these methods lack the stability because they used to be interfered by environments and other objective factors due to their own limitations, which lead to a few use.Traditional assessments, such as used to differentiating muscle fatigue in accordance with time domain index, such as average Electromyography(AEMG),Integral EMG(iEMG), and Root mean square(RMS) and frequency domain index such as Median frequency(MF), Average power frequency(MPF) [11] [12]. These traditional methods consider that EMG signals are linear, stable or stationary or piecewise stationary, but the feature of sEMG is highly nonlinear and the signal form is random and unstable, which indicates the traditional linear spectrum is insufficient to reflect the essential feature of signal changes [13] [14]. Therefore, applying non-linear dynamic analysis method can extract more effective and stable features of signals in terms of analyzing sEMG, which helps improve the recognition rate of EMG signals [15].

Wavelet packet entropy is a newer non-linear index, because it is an entropy value calculated from a sequence of signals that wavelet packet is decomposed [16] [17]. It shows a good resolution in the domain of time and frequency and has become an effective tool for non-stationary signals. The energy distribution has been widely used in processing and analyzing EEG signals: the study of Zhao Li et al. proved that Mahalanobis distance can classify and distinguish the feature vectors of the combination of wavelet packet energy and wavelet entropy, which contributes to the classification of motor imagery signals whose average correct rate reaches by $84.3 \%$; Shen Min-fen et al. revealed that more complex are components of the signal frequency, the closer is the energy of relative wavelet packet, and the larger is the value of wavelet packet entropy, which thoroughly explained that wavelet packet entropy can act excellent in reflecting the the complexity of EEG signal rhythm[18]. However, the wavelet packet entropy has been barely applied in estimation of muscle fatigue in the related research. [19] In order to test the effectivity of wavelet packet entropy in the process of rehabilitation, this research used the experiment of multi-channel sMEG to record muscle fatigue illustrated by wavelet packet energy, and analyzed experimental statistics for further testing and verifying [20]. 


\section{Materials and methods}

\section{1. paticipants}

In this experiment, in order to reduce the influence of individual differences on the results of later data analysis, five healthy young people were randomly selected as subjects from graduate students in universities such as Zhejiang Sci-Tech University. The subjects have no professional sports training experience, and they are all right-handed, have good physical health, and have no history of muscle damage. At the same time, to prevent the subjects from excessive exercise before the experiment, the reliability and validity of the data collected in the experiment will be prevented. Impact, did not engage in vigorous exercise 48 hours before the start of the formal experiment, no muscle fatigue symptoms.

\subsection{Experimental protocol}

To establish the experimental model closer to the actual situation while ensuring the validity of the experimental data, the researchers selected a grasping action that can effectively help the patient with post-recovery.Medical research shows that the brachioradialis muscle in the upper limbs of the human body is the force exerting force when griping, and the triceps brachii is the main force exerting muscle when the arm does stretching. Therefore, this experiment will collect the sEMG of the brachioradialis and triceps to study the changes in the characteristic parameters of the sEMG during muscle fatigue.

At present, because there is no specific general standard for muscle fatigue, the experiment evaluates the fatigue state of the tested muscles through subjective feelings and experience. Considering the time validity of the transition from the experiment preparation stage to the beginning stage, the researchers estimated the actual fatigue degree by intercepting the time point. Specifically, when the subject starts to exert force for 2 seconds, the degree of muscle fatigue is $0 \%$; When the subject continues to perform the designated task until exhausted and cannot continue to perform the task with external encouragement, the degree of muscle fatigue is $100 \%$.

This experiment takes the brachioradialis muscle as an example to study the relationship between the changes of electromyographic characteristic parameters and the degree of muscle fatigue during the muscle fatigue process. Brachioradialis's periodic contraction experiment is the primary test in the fatigue load test process. Brachioradialis constant force contraction experiment is used to study the influence of force mode on the change of characteristic parameters. Each experiment process includes one maximum voluntary contraction force test and one fatigue test. The fatigue test includes a periodic contraction fatigue test of the brachioradialis muscle and a fatigue test of brachioradialis muscle constant contraction fatigue experiment.

\subsection{Date collection}

Before starting the formal experiment, the researchers demonstrate the overall experimental process and specific practical actions to the subjects. After the subjects are familiar with the process and actions, the researchers determine the subjects' maximum voluntary contraction(MVC, Maximal Voluntary Contraction) in different postures, and wait for the subjects to rest fully and their muscles to return to normal conditions before the formal experiment. In each experiment, the researchers use a video recorder to record 
the entire experiment process. After the subjects completed a fatigue experiment, they needed to rest for one to two days to ensure enough muscle rest time to recover their physical strength to avoid the impact of residual fatigue in the two experiments.

In the fatigue experiment, the setting of the subject's force references the maximum voluntary contraction force $(\% \mathrm{MVC})$. The weight loading of different subjects is set according to their respective maximum voluntary contraction force. During the experiment, the researchers used the commercial grip strength measuring instrument EH101 to obtain the maximum voluntary contraction force parameters. The average value of three measurements is used as the calculation to make the data more accurate, as shown in Figure 1. The sEMG is acquired through a 10-channel Motion Lab System (MA300) equipment, and the sampling frequency is $1000 \mathrm{~Hz}$.

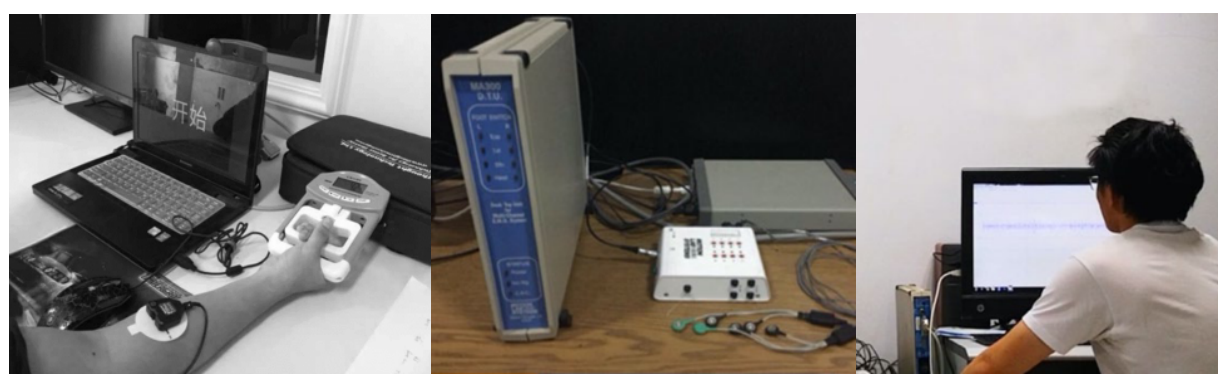

Figure 1. The testing process of the contraction fatigue of brachioradialis

The subject is sitting in front of the test bench, with the right arm flat on a horizontal surface, and holding the grip strength tester with a constant force of $20 \%$ MVC until it can no longer hold. After the experiment of constant force contraction of the brachioradialis muscle, the subject rested for 20-30 minutes to ensure that the hand grasping returned to normal state. The subject is sitting in front of the test bench, with the right arm flat on a horizontal surface and periodically grasping the grip tester. Each gripping cycle lasts for $5 \mathrm{~s}$ (including $3 \mathrm{~s}$ exertion and $2 \mathrm{~s}$ rest). Each time the subject exerted force, they held the dynamometer to the end and then relaxed. The subject continued to exert force in this way periodically until they were unable to persist after exhaustion.

\subsection{Algorithm explanation}

$\mathrm{H}$ is set in the $\mathrm{j}$-th frequency band of the frequency window in the subspace of. In the decomposition of wavelet packet entropy, $\mathrm{J}$ frequency band is further divided into frequency bands:

$$
H_{j}^{k, m}, m=0,1,2, A, 2^{k}-1
$$

All sub-frequency bands have been composed $H_{j}$ :

$$
Y_{m=0}^{2^{k}-1} H_{j}^{k, m}=H_{j}
$$

Therefore, the decomposition of wavelet packet entropy improves the resolution of high frequency component. Afterwards, the sub-signals in the i-th subspace can be reconstituted below:

$$
S_{j}^{i}(t)=\sum_{k} D_{k}^{j, i} \Psi_{j, k}(t)
$$

The energy of the reconstructed signal in each subspace is 


$$
E_{j}=\sum_{k}\left|D_{k}^{i, j}\right|^{2}
$$

According to the law of conservation of energy, total signal energy $E$ equals the sum of the energy of each subspace. The distribution of probability density of energy, in each frequency band is obtained by the energy in the subspace which is normalized the total energy of the $\operatorname{signal}\left(P_{j}=E_{j} / E\right)$, which is called the relative wavelet energy. It can quantify the energy distribution of the signal in each frequency band.

Information entropy can test the level of unknown and estimate the complexity of random signals.Based on the energy distribution of decomposition coefficients of wavelet packet and information entropy, the wavelet packet entropy can be defined as:

$$
\mathrm{WE}=-\sum_{i=1}^{n} P_{i} \ln P_{i}
$$

Wavelet packet entropy can measure ordered and disordered information in the quantitative way. If sEMG energy gather in a sub-frequency band, WE will be 0 , which means it is ordered. Conversely, the sEMG energy is randomly scattered across the subbands, meaning it is a sign of disorder.

\section{Rexults and discussions}

\subsection{Date processing}

Experiment statistics are collected by Motion Lab System(MA300) of 10 channel. The software, Matl -lab, is used for offline processing and analyzing collected signals. The original electrical muscle signals were pre-processed by an IIR band-pass filter at 20-450 $\mathrm{HZ}$ and subsequently segmented based on the duration of the single contraction. Meanwhile, in the case of signal interception, it is used to intercepting signal periods at the $50 \%$ of the signal, which protects overlapped signal features from losing and ensuring the validity of signals. As showed in Figure 2, for reducing errors, the first and last contraction are abandoned in the process of analysis, and using multi-level segmentation to the signal band after signal decomposing. Additionally, reconstruct the sub-signals in the subspace and normalize the signal energy of each band, and then calculate the wavelet packet entropy, and finally present the image by linear fitting the wavelet packet entropy of each segment. As illustrated in Figure 3, this method can be predicted muscle fatigue in a simple and direct way.
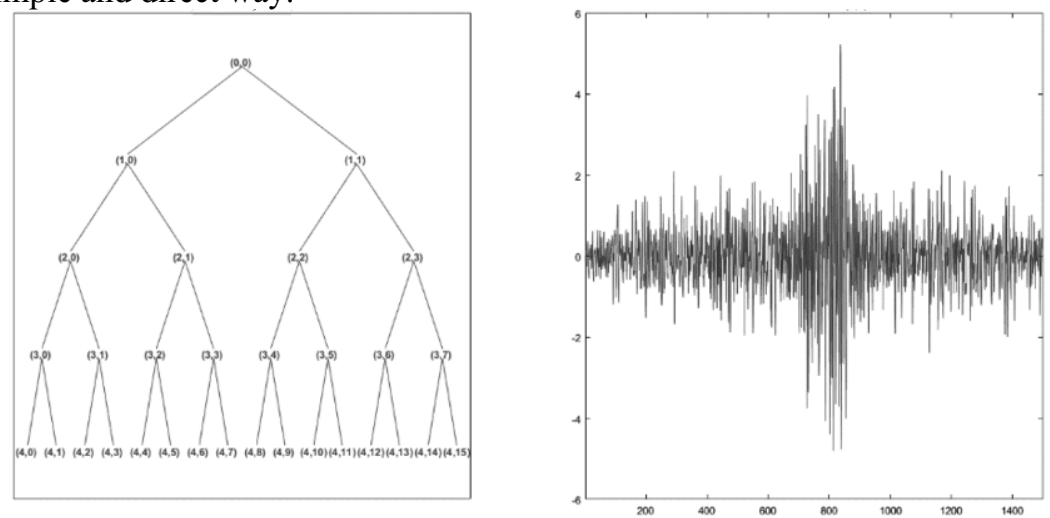

Figure 2. The subspace reconstruction image of sEMG decomposed by wavelet packet 


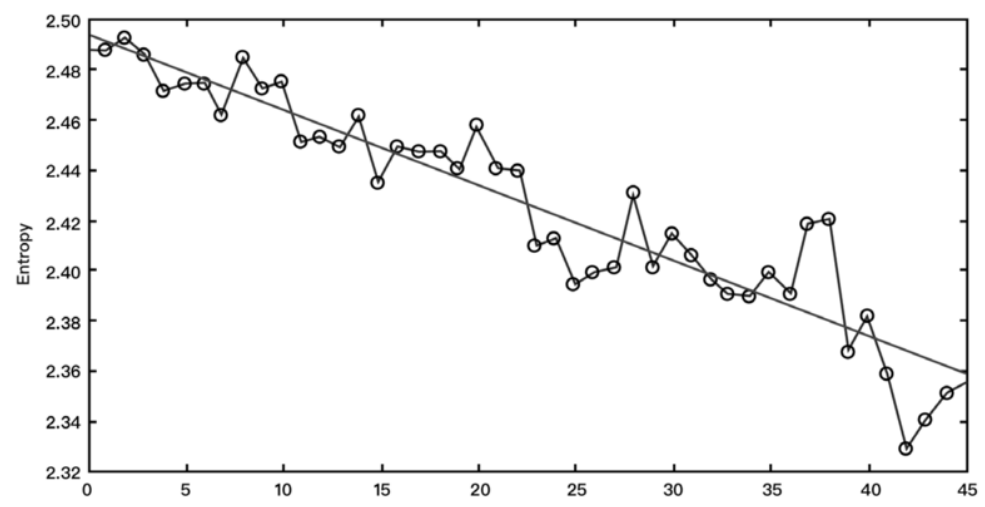

Figure 3. Time distribution chart of wavelet packet entropy

\subsection{Analysis of Wavelet packet entropy energy}

Figure 4 describes that how the percentage of sEMG energy of wavelet packet transforms as subspace frequency band changes, during the first 30 s experiment of constant force contraction and periodic force contraction. Figure 5 shows the transformation of energy in the last 30s. The transformation in different subspaces tends to be consistent when decomposing wavelet packet, which means that the wavelet packet energy at the low frequency band is constantly higher than that at the high frequency band, regardless of constant force contraction or periodic force contraction. As the muscles become progressively fatigued, it is witnessed an increase in the proportion of wavelet packet energy at the lower frequency bands in the sEMB, while the energy of wavelet packets at the higher frequency's decreases, which means the signals move from high to low frequency. This is same as the generally accepted view that spectrum compresses to the left while fatigue increasing.

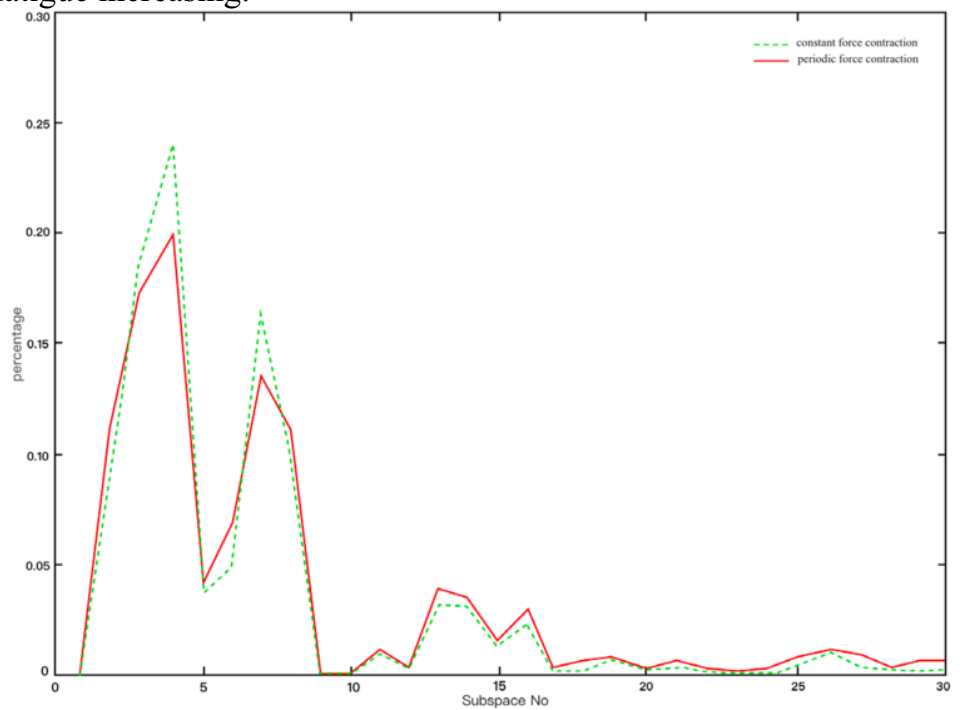

Figure4. Time distribution chart of wavelet packet entropy 


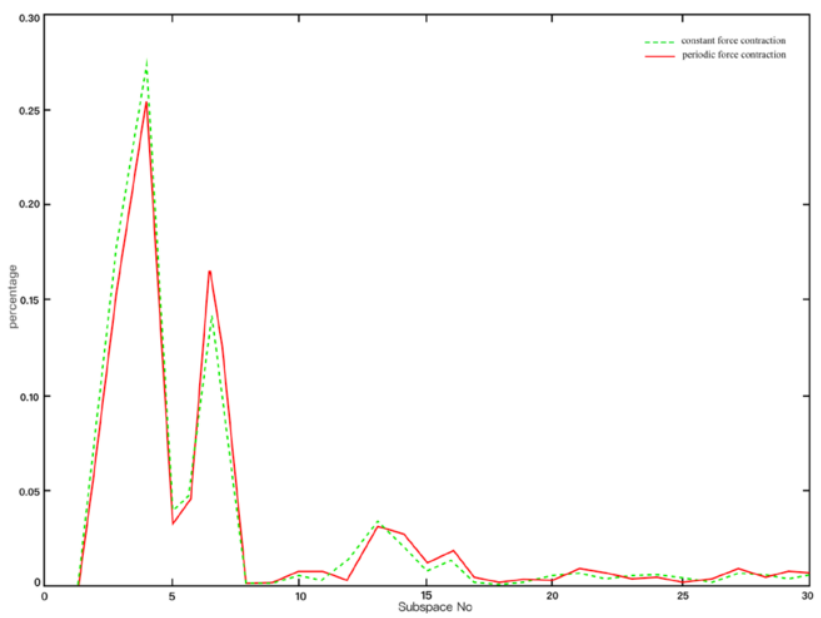

Figure5. Time distribution chart of wavelet packet entropy

Biological studies have stated that the tissue between the motor unit and the surface electrodes has a low-pass filtering effect on the motor unit potential, that is the basic component of SEMG. Muscle inconsistently contracts in the different movement, which results in the energy of SEMG signals distribute diversely in different frequency bands.

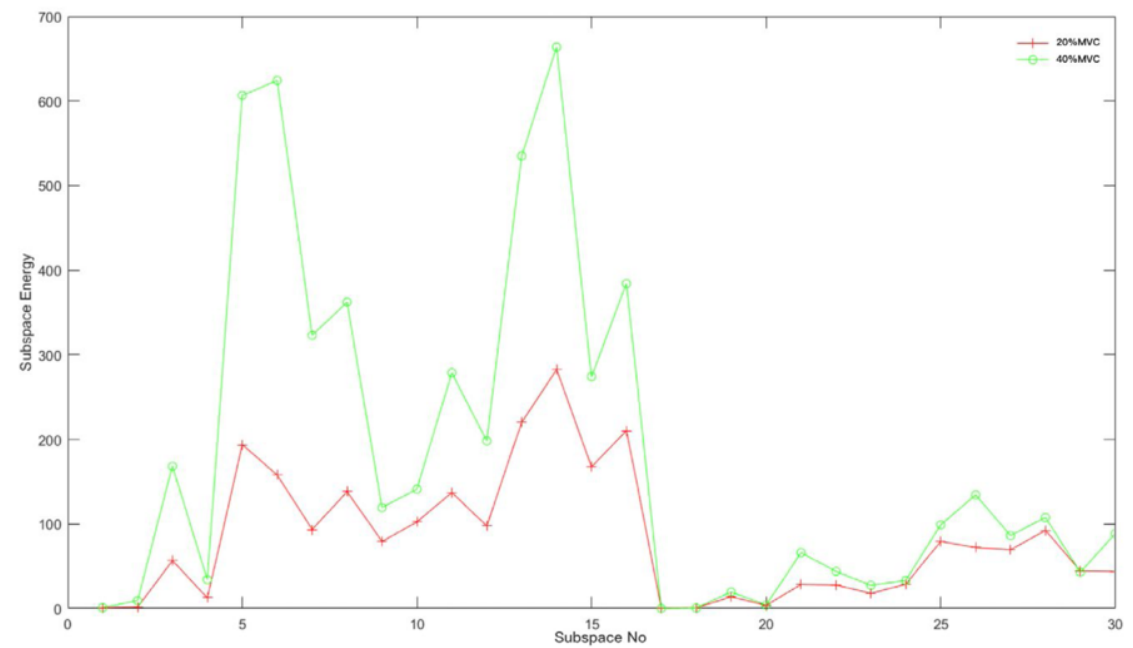

Figure6. the distribution map of wavelet packet in subspace when brachioradialis exercise with different muscle strength

\subsection{Analysis of the relationship between wavelet packet entropy and muscle fatigue}

As Figure 7 and Figure 8 shows, while the level of muscle fatigue increasing, there is a decrease in the wavelet packet entropy, regardless in the case of constant force contraction or periodic force contraction. It can be deduced that there is a synergy between wavelet packet entropy and muscle fatigue, meaning the value of wavelet packet entropy can be used as an important indicator to assess the muscle fatigue. 


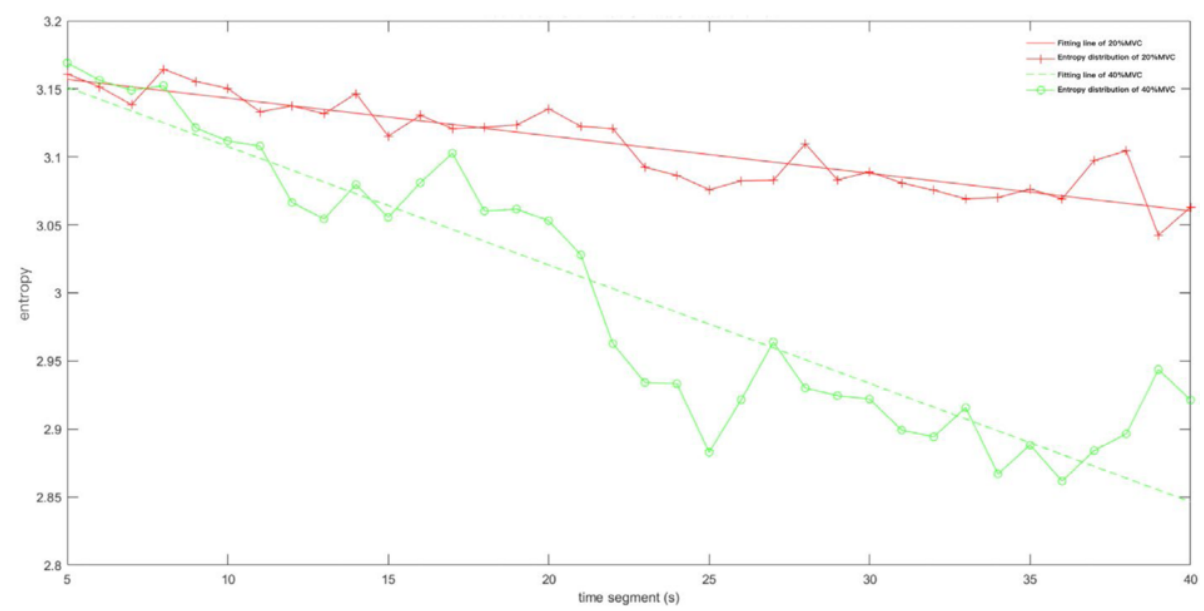

Figure 7. The distribution chart of wavelet packet entropy with time for different muscle force

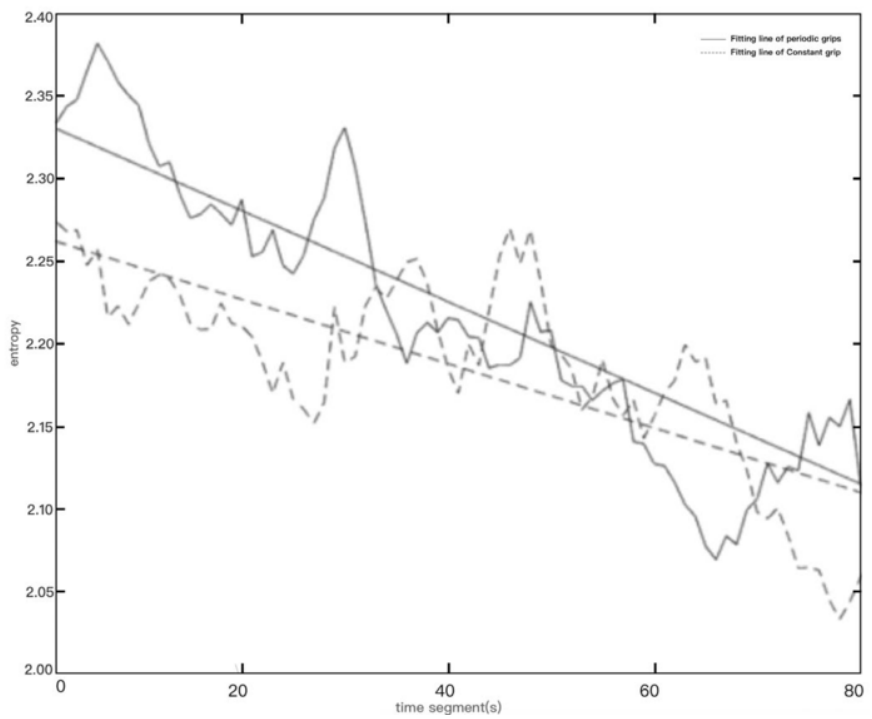

Figure 8. Comparison of wavelet packet entropy distribution in the brachioradialis experiment of constant force contraction or periodic force contraction (Right)

In the Figure 9, it is obvious that, with the training of an upper limb, there is a consistent decreasing trend in the value of wavelet packet entropy. This offers a reliable reference for the assessment of muscle fatigue, although it is difficult to test out the precise critical values to explain the muscle fatigue due to the huge variability among individuals. 


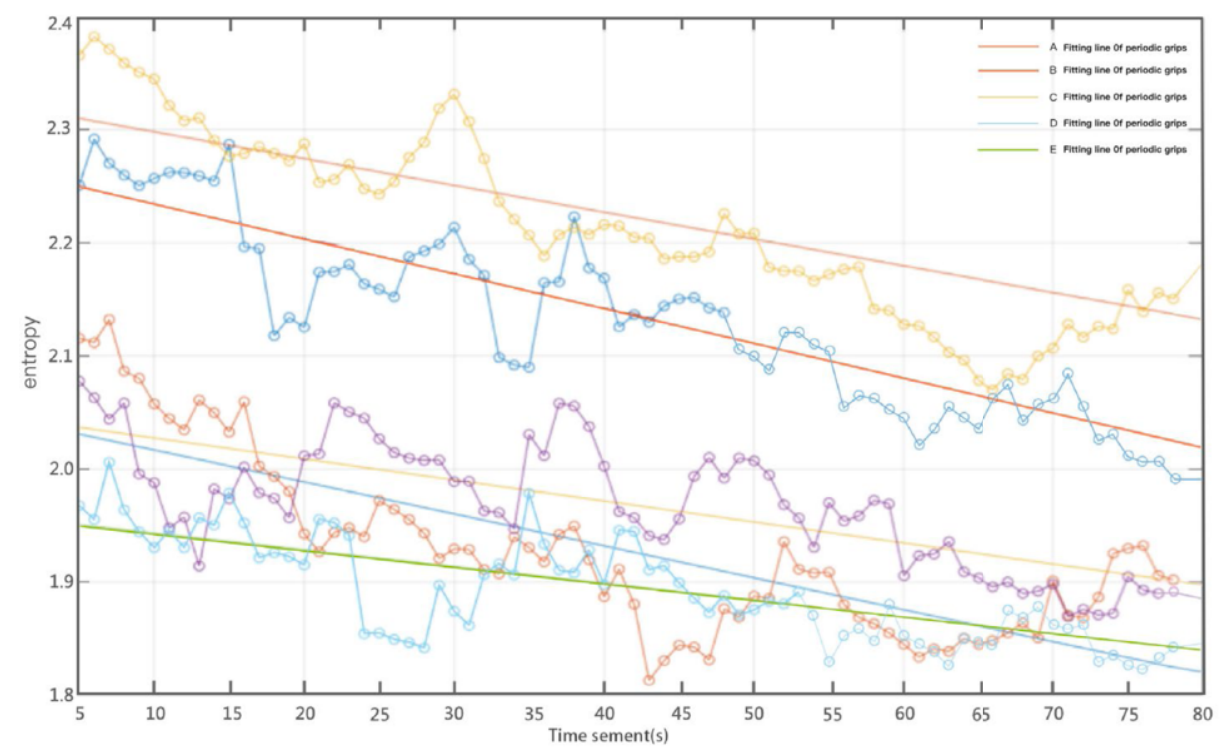

Figure 9. Time distribution chart of wavelet packet entropy when different participants test periodic force contraction

For a more effective instruction for rehabilitation training, we analyze the data by min-max normalization and observe the data pattern. The conversion function is as follows:

Max is the maximum value of the sample data and min is the minimum value of the sample data. Based on normalization and reference to current domestic and international literature on muscle fatigue, the initial domain value of muscle fatigue is defined as 0.2 . When this value is temporarily less than or equal to 0.2 , it indicates that the muscles are fatigued. The speed of training needs to be slowed down and the load of rehabilitation training should be reduced.

\section{Conclusion}

This research proposes that wavelet packet entropy can be regarded as the measurement to test the level of muscle fatigue. In addition, this research focuses on experimenting the relationship between the energy variation of wavelet packet entropy and the degree of muscle fatigue when testing the muscle contraction by the sEMG signals of brachioradialis. The result of experiments indicated that wavelet packet entropy allows efficient and fast qualitative analysis of system complexity. High accuracy, instant reaction, stronger consistency and reliability could be the key reference indicators for assessing muscle fatigue which is meaningful for the achievement of the real-time monitoring and clinical research of bioelectrical signals. However, due to the large individual variability, less participants has been invited in the experiments, which could result in some errors in the result, which, therefore, needs further research. 


\section{References}

[1] K. Veer. A technique for classification and decomposition of muscle signal for control of myoelectric prostheses based on wavelet statistical classifier. Measurement. 2015; 60:283-291.

[2] Marco Y. Pang, PhD, Jocelyn E. Harris, MSc, Janice J. Eng, PhD. A Community-Based Upper-Extremity Group Exercise Program Improves Motor Function and Performance of Functional Activities in Chronic Stroke: A Randomized Controlled Trial.ArchPhys Med Rehabil.2006,87(1):1-9.

[3] Dimitrov GV, Arabadzhiev TI, Mileva KN, Bowtell JL, Crichton N, Dimitrova NA. Muscle fatigue during dynamic contractions assess-ed by new spectral indices. Med Sci Sports Exerc 2006;38(11):19719.

[4] Song JH, Jung JW, Bien Z. Robust EMG pattern recognition to muscular fatigue effect for humanmachine interaction. Lect Notes Comput Sc. 2006; 4293:1190-9.

[5] Allen DG, Lamb GD, Westerblad H. Skeletal muscle fatigue: Cellular mechanisms. Physiol Rev. 2008; $88: 287-332$.

[6] Reaz MBI, Hussain MS, Mohd-Yasin F. Techniques of EMG signal analysis: detection, processing, classification and applications. Biol Proced Online. 2006; 8(1):11-35.

[7] Y. Zeng, Y. YangZ, W. Cao, C.M Xia.Hand-motionPatterns Recognition based on Mechanomyographic Signal Analysis.2009 International Conference on Future BioMedical Information Engineering .2009; pp. 21-24.

[8] H.J. Ding, Q. He, L. Zeng, Y.J. Zhou, Shen Minmin, Guo Dan.Motion intent recognition of individual fingers based on mechanomyogram. Pattern Recogn Lett, 2017;88:41-48.

[9] Tong S, Bezerianos A, Paul J, etal. Nonextensive entropy measure of EEG following brain injury from cardiac arrest source [ J]. Physica A: Statistical Mechanics and its Applications,2002, 305(3-4): 619-628.

[10] Gonz a'lez-Izal M, Rodríguez-Carreño I, Malanda A, Mallor-Giménez F, Navar-ro-Amézqueta I, Gorostiaga EM, et al. sEMG wavelet-based indices predicts muscle power loss during dynamic contractions. J Electromyogr Kinesiol .2010;20:106-107.

[11] Xie H-B, Guo J-Y, Zheng Y-P. Fuzzy approximate entropy analy-sis of chaotic and natural complex systems: detecting muscle fatigue using electromyography signals. Ann Biomed Eng 2010;38(4):148396.

[12] B. Besselink, K.H. Johansson.String stability and a delay-based spacing policy for vehicle platoons subject to disturbances.IEEE Transactions on Automatic Control, 2017;62 (9):4376-4391.

[13] Guo S, Pang M, Gao B, Hirata H, Ishihara H. Comparison of sEMG-based feature extraction and motion classification methods for upper-limb movement. Sensors (Basel) 2015;15 (4):9022-38.

[14] Lascurain-Aguirrebeña I, Newham DJ, Irazusta J, Seco J, Critchley DJ. Reliability of a method to measure neck surface electromyography, kinematics, and pain occurrence in participants with neck pain. J Manipul Physiol Therap. 2018;41(5):413-24.

[15] Liang S, Wu Y, Chen J, Zhang L. Identification of gesture based on combination of raw sEMG and sEMG envelope using supervised learning and univariate feature selection. J Bionic Eng. 2019; 16:647-62.

[16] Y. Gu, K. Dremstrup, D. Farina.Single-trial discrimination of type and speed of wrist movements from EEG recordings.Clin.Neurophysio, 2009;120 (8) :1596-1600.

[17] T. Platz, I.H. Kim, H. Pintschovius, T. Winter, A. Kieselbach, K. Villringer, K.H. Mauritz.Multimodal EEG analysis in man suggests impairment-specific changes in movement-related electric brain activity after stroke.Brain. 2000; 123 (12): 2475-2490.

[18] M. Burnley.Estimation of critical torque using intermittent isometric maximal voluntary contractions of the quadriceps in humans.J. Appl. Physiol. Mar 2009; 106 (3): 975-983.

[19] L.H. Chung, D.M. Callahan, J.A. Kent-Braun.Age-related resistance to skeletal muscle fatigue is preserved during ischemia.J. Appl. Physiol, Nov 2007;103 (5) :1628-1635.

[20] L.A. Frey-Law, J.M. Looft, J. Heitsman.A three-compartment muscle fatigue model accurately predicts joint-specific maximum endurance times for sustained isometric tasks.J.Biomech. Jun2012;45 (10): 1803-1808. 